\title{
Highly Reactive Cationic Molybdenum Alkylidyne N-Heterocyclic Carbene Catalysts for Alkyne Metathesis
}

Jonas Groos $^{ \pm}$, Philipp M. Hauser ${ }^{ \pm}$, Maximilian Koy ${ }^{ \pm}$, Wolfgang Frey ${ }^{\ddagger}$, and Michael R. Buchmeiser ${ }^{ \pm}, *$

${ }^{ \pm}$Institute of Polymer Chemistry and ${ }^{\neq}$Institute of Organic Chemistry, University of Stuttgart, Pfaffenwaldring 55, 70569 Stuttgart, Germany

\section{Contents}

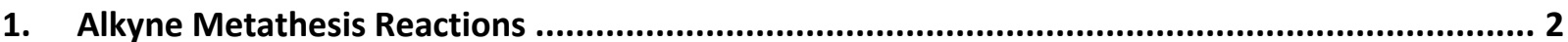

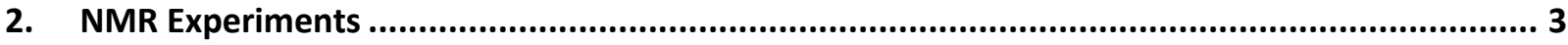

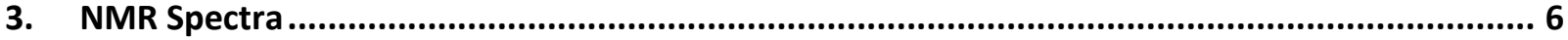

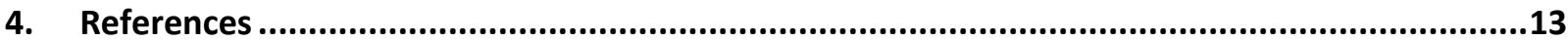




\section{Alkyne Metathesis Reactions}

Table S1: Turnover numbers (TONs) and conversion in alkyne self-metathesis of substrates S1-S6 at a concentration of $130 \mathrm{mM}$.

\begin{tabular}{|c|c|c|c|c|c|c|}
\hline Entry & Catalyst & $\begin{array}{c}\text { Substrate: } \\
\text { Catalyst }\end{array}$ & Product & $\begin{array}{c}\text { Time } \\
{[\mathrm{h}]}\end{array}$ & TON & $\begin{array}{c}\text { Conversion } \\
{[\%]}\end{array}$ \\
\hline 1 & Mo4 & 1000 & & 3 & 800 & 80 \\
\hline 2 & Mo5 & 1000 & s1 & 3 & 780 & 78 \\
\hline 3 & Mo4 & 1000 & & 3 & 980 & 98 \\
\hline 4 & Mo5 & 1000 & & 3 & 970 & 97 \\
\hline 5 & Mo4 & 1000 & & 3 & $\overline{970}$ & 97 \\
\hline 6 & Mo5 & 1000 & & 3 & 960 & 96 \\
\hline 7 & $\overline{\text { Mo4 }}$ & 1000 & & 3 & 840 & 84 \\
\hline 8 & Mo5 & 1000 & & 3 & 780 & 78 \\
\hline 9 & $\overline{M o 4}$ & 1000 & & 3 & $\overline{970}$ & 97 \\
\hline 10 & Mo5 & 1000 & S5 & 3 & 970 & 97 \\
\hline 11 & $\overline{M o 4}$ & 1000 & & 3 & 790 & 79 \\
\hline 12 & Mo5 & 1000 & & 3 & 670 & 67 \\
\hline
\end{tabular}

Solvent: 1,2-dichlorobenzene; internal standard: tBu-benzene; temperature: $35^{\circ} \mathrm{C}$; with the addition of ground $5 \AA$ molecular sieves as 2-butyne scavenger. ${ }^{1}$

Table S2: Turnover numbers (TONs) and conversion in alkyne self-metathesis of substrates S1-S6 with complex Mo6 after three hours at a concentration of $660 \mathrm{mM}$ using a substrate : catalyst ratio of $20000: 1$.

\begin{tabular}{ccccc}
\hline Entry & Substrate & TON $_{3 \mathbf{h}}$ & Time [h] & Conversion [\%] \\
\hline 1 & $\mathbf{S 1}$ & 4400 & 3 & 22 \\
\hline 2 & $\mathbf{S 2}$ & 10600 & 3 & 53 \\
\hline 3 & $\mathbf{S 3}$ & 8900 & 3 & 45 \\
\hline 4 & $\mathbf{S 4}$ & 9200 & 3 & 44 \\
\hline 5 & $\mathbf{S 5}$ & 8700 & 3 & 34
\end{tabular}

Solvent: 1,2-dichlorobenzene; internal standard: $t$ Bu-benzene; temperature: $35^{\circ} \mathrm{C}$; with the addition of ground $5 \AA$ molecular sieves as 2-butyne scavenger. ${ }^{1}$ 


\section{NMR Experiments}

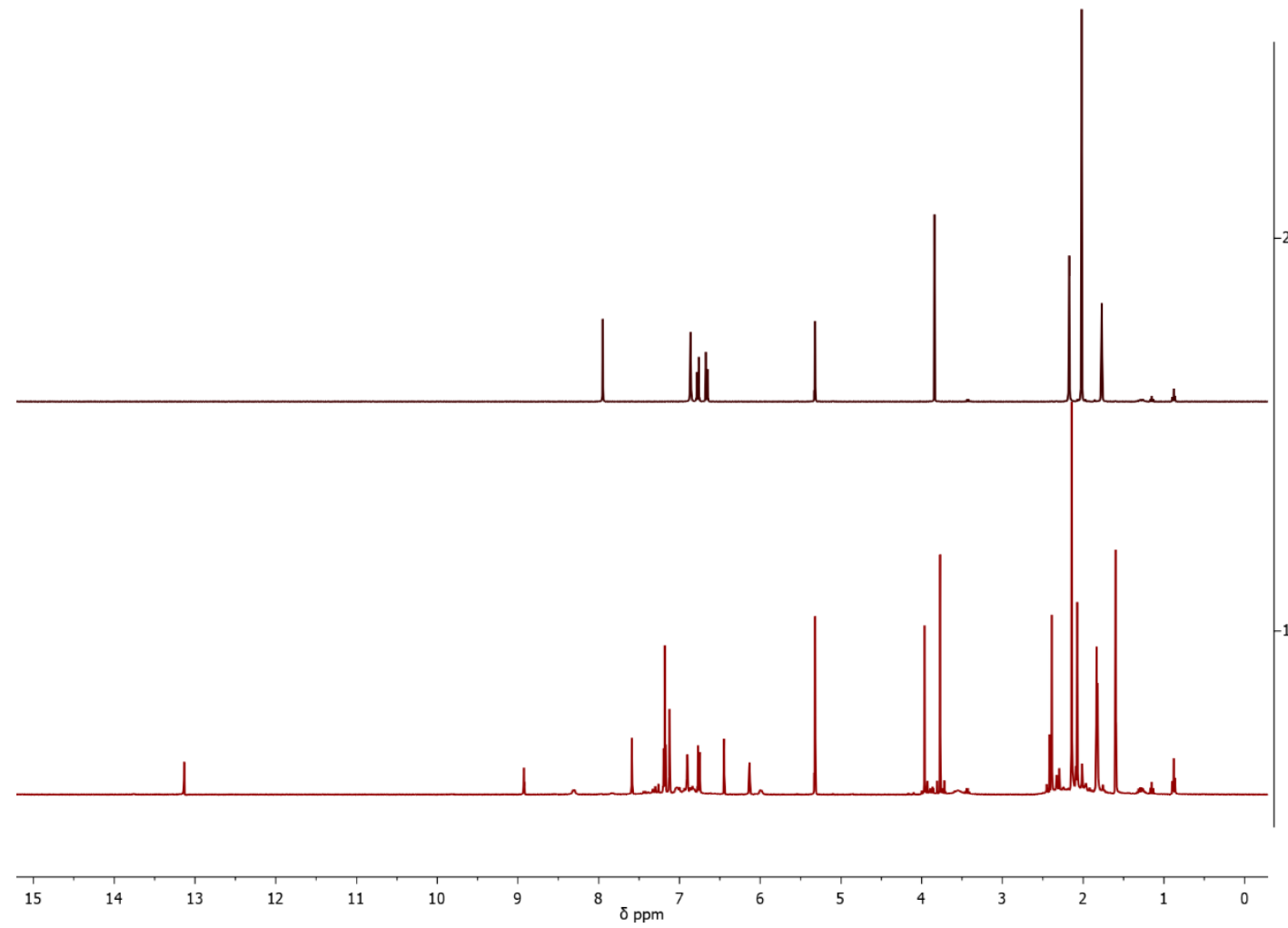

Figure S1: ${ }^{1} \mathrm{H}$ NMR $\left(400 \mathrm{MHz}, \mathrm{CD}_{2} \mathrm{Cl}_{2}\right.$ ) spectrum of Mo4 before (top) and after (bottom) exposure to air for 24 hours.

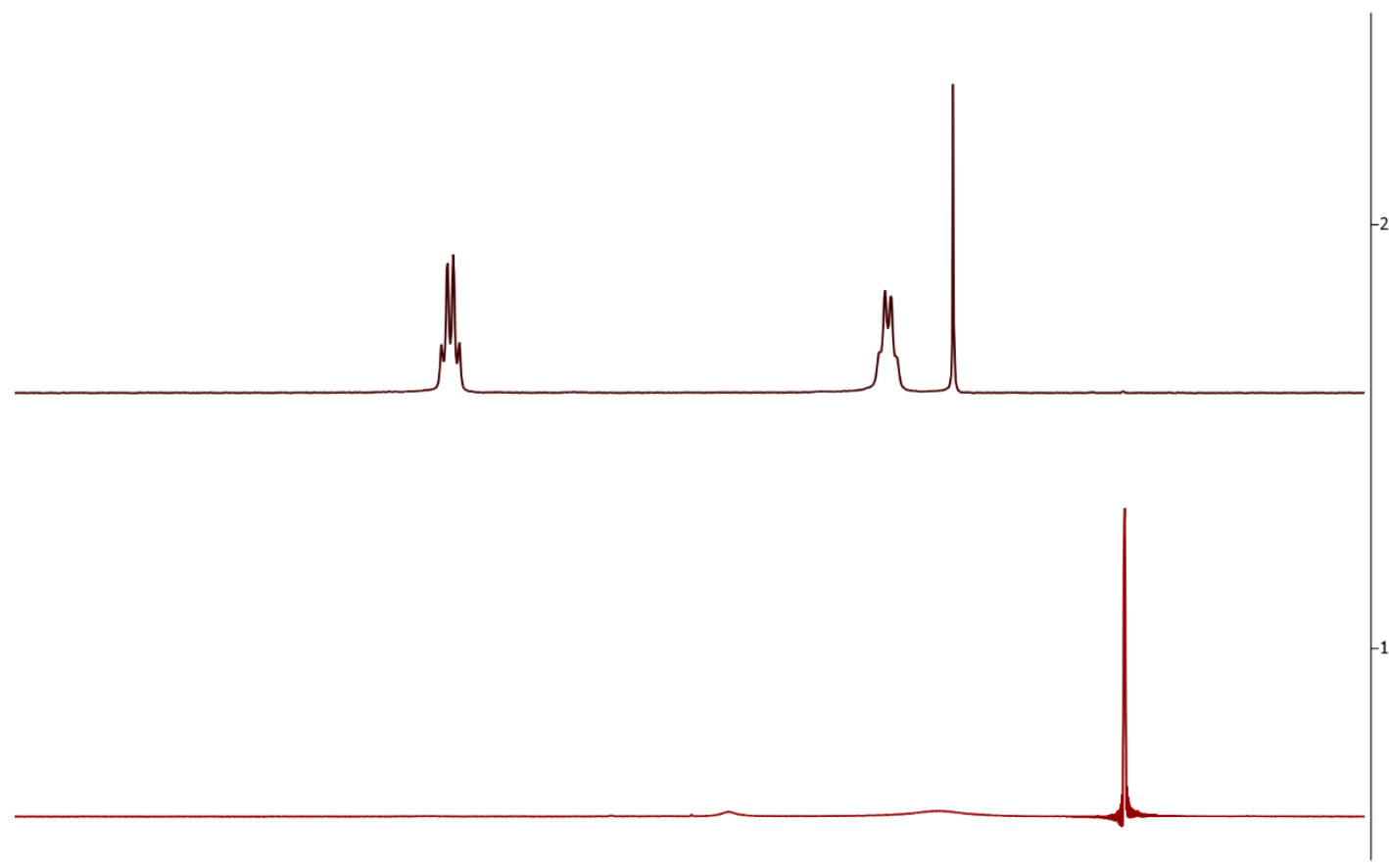

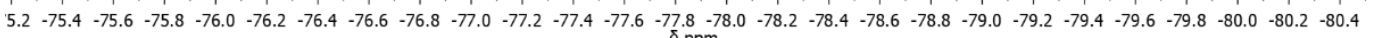

Figure S2: ${ }^{19} \mathrm{~F} \mathrm{NMR} \mathrm{(375} \mathrm{MHz,} \mathrm{CD}_{2} \mathrm{Cl}_{2}$ ) spectrum of Mo4 before (top) and after (bottom) exposure to air for 24 hours. 


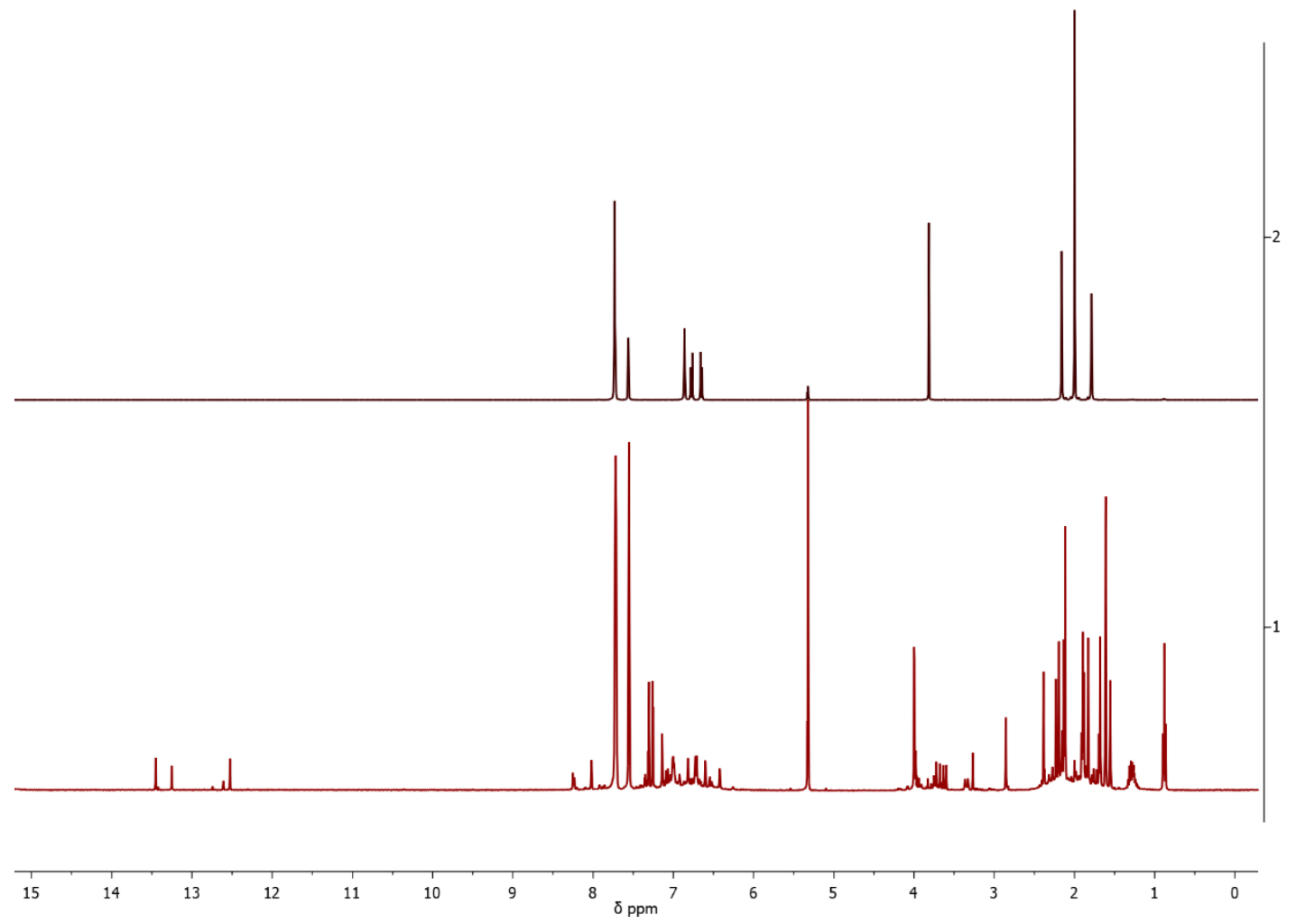

Figure S3: ${ }^{1} \mathrm{H}$ NMR (400 MHz, $\mathrm{CD}_{2} \mathrm{Cl}_{2}$ ) spectrum of Mo6 before (top) and after (bottom) exposure to air for 24 hours.
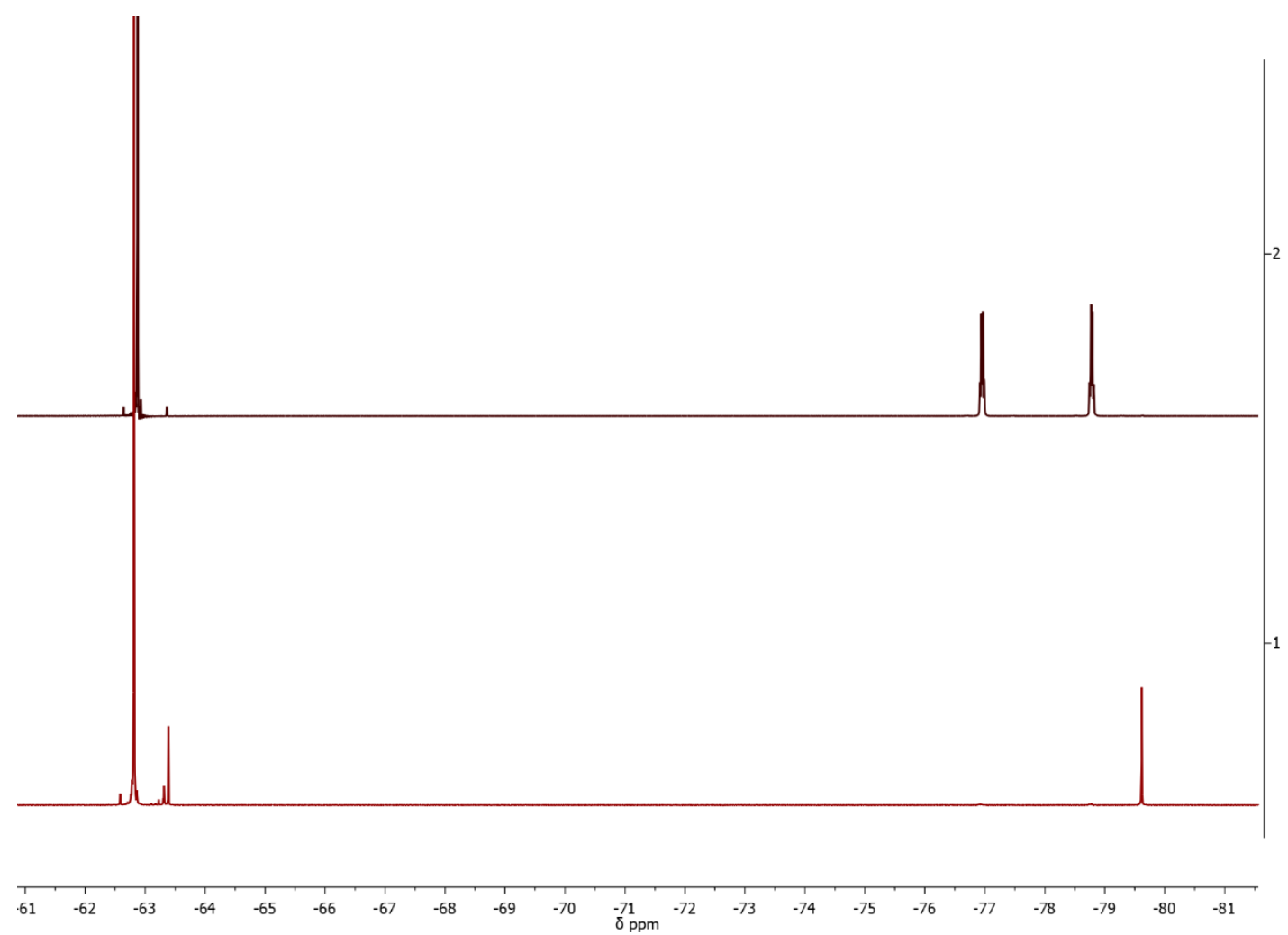

Figure S4: ${ }^{19} \mathrm{~F} \mathrm{NMR} \mathrm{(375} \mathrm{MHz,} \mathrm{CD}_{2} \mathrm{Cl}_{2}$ ) spectrum of Mo6 before (top) and after (bottom) exposure to air for 24 hours. 


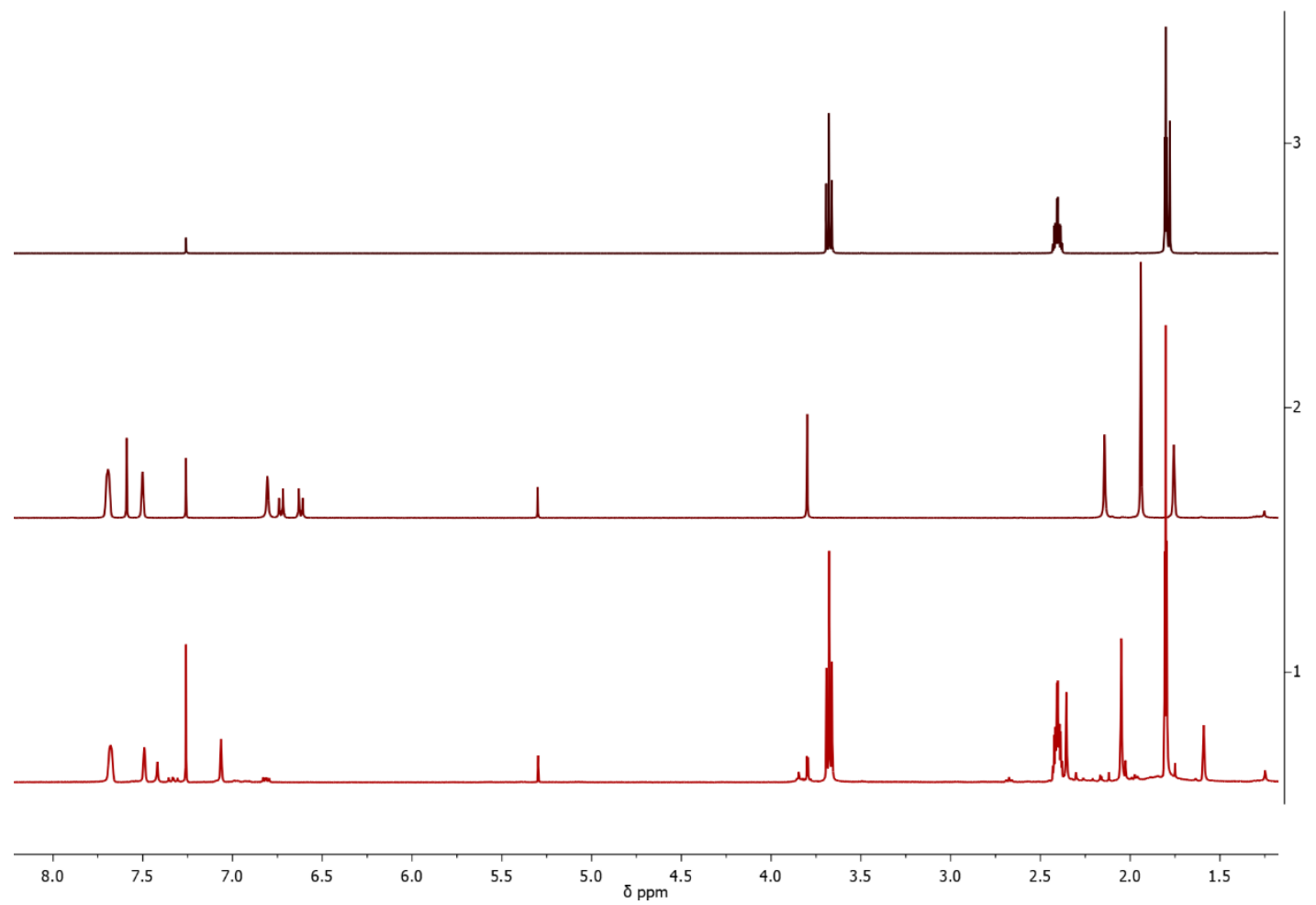

Figure S5: ${ }^{1} \mathrm{H}$ NMR $\left(400 \mathrm{MHz}, \mathrm{CDCl}_{3}\right)$ spectrum of Mo6 before (middle) and after (bottom) reaction with 10 equivalents of 3-pentyn-1-ol as well as spectrum of 3-pentyn-1-ol (top).

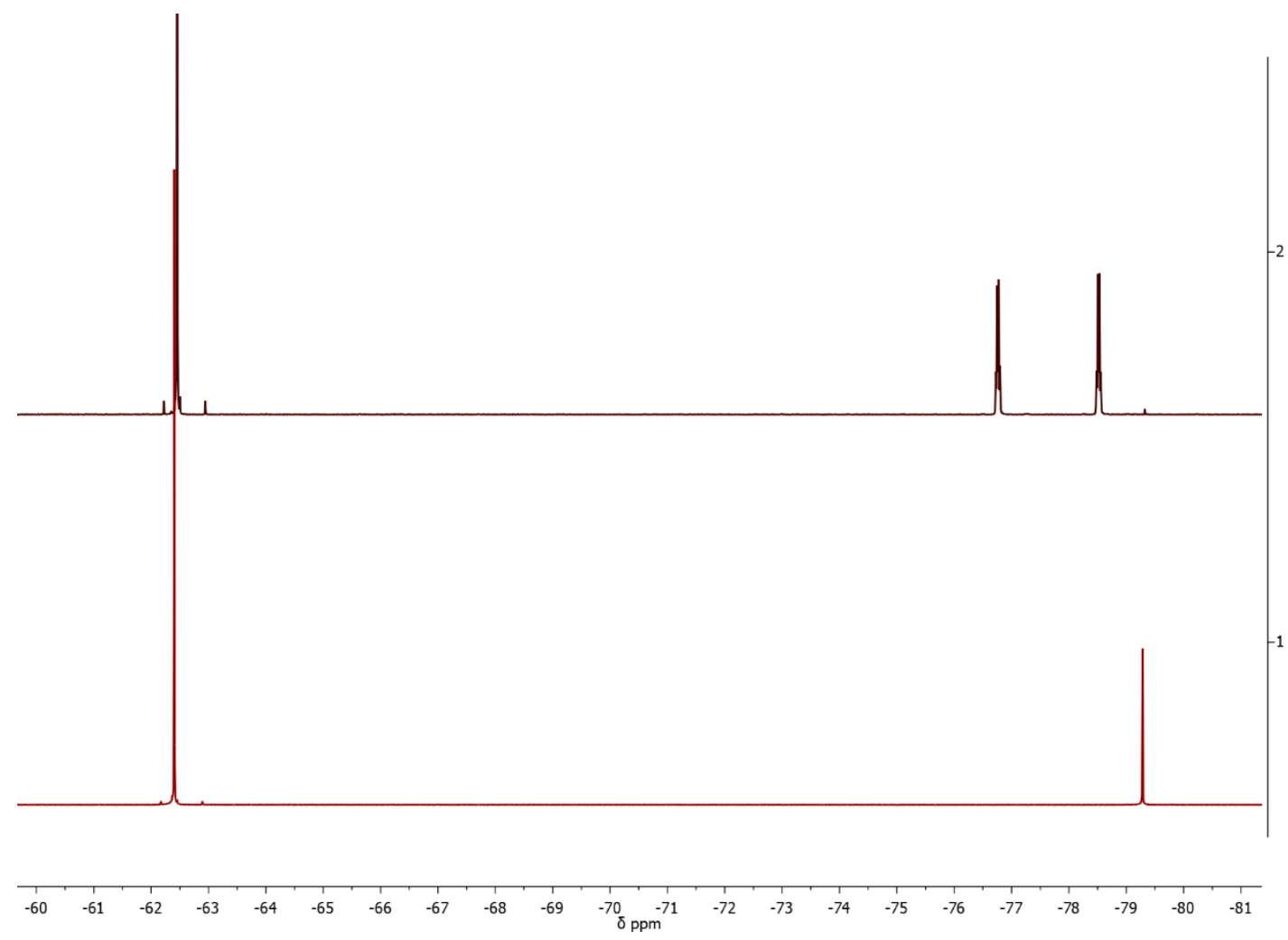

Figure S6: $\left.{ }^{19} \mathrm{~F} \mathrm{NMR} \mathrm{(375} \mathrm{MHz,} \mathrm{CD}_{2} \mathrm{Cl}_{2}\right)$ spectrum of Mo6 before (top) and after (bottom) reaction with 10 equivalents of 3-pentyn-1-ol. 


\section{NMR Spectra}

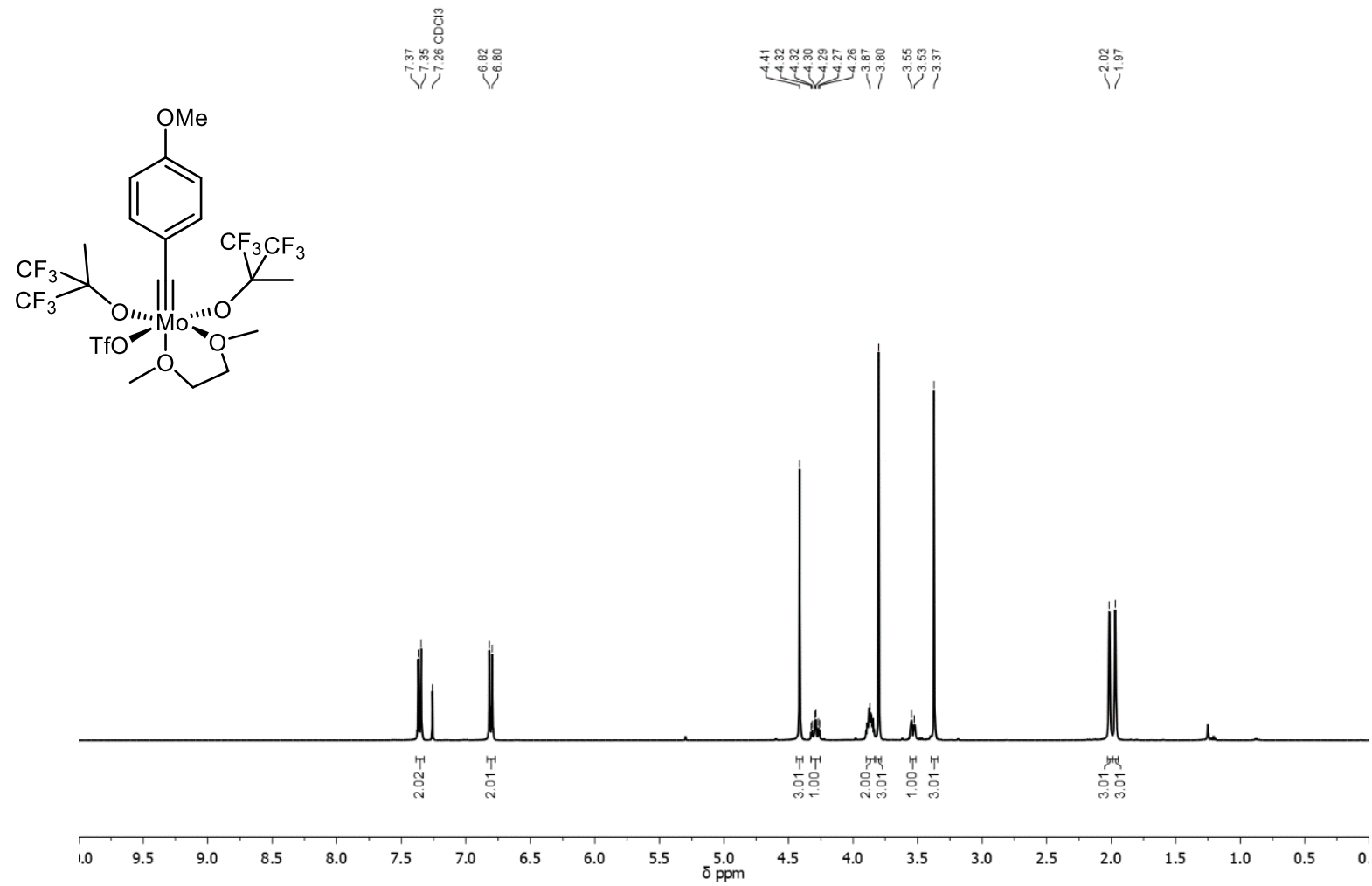

Figure S7: ${ }^{1} \mathrm{H}$ NMR (400 MHz, $\mathrm{CDCl}_{3}$ ) spectrum of $\mathbf{M o 2}$.

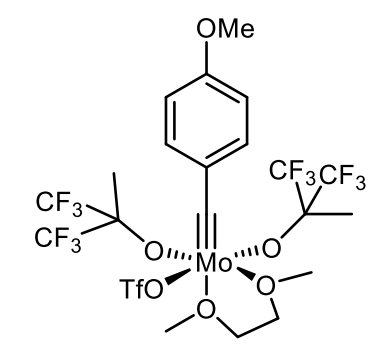

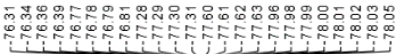
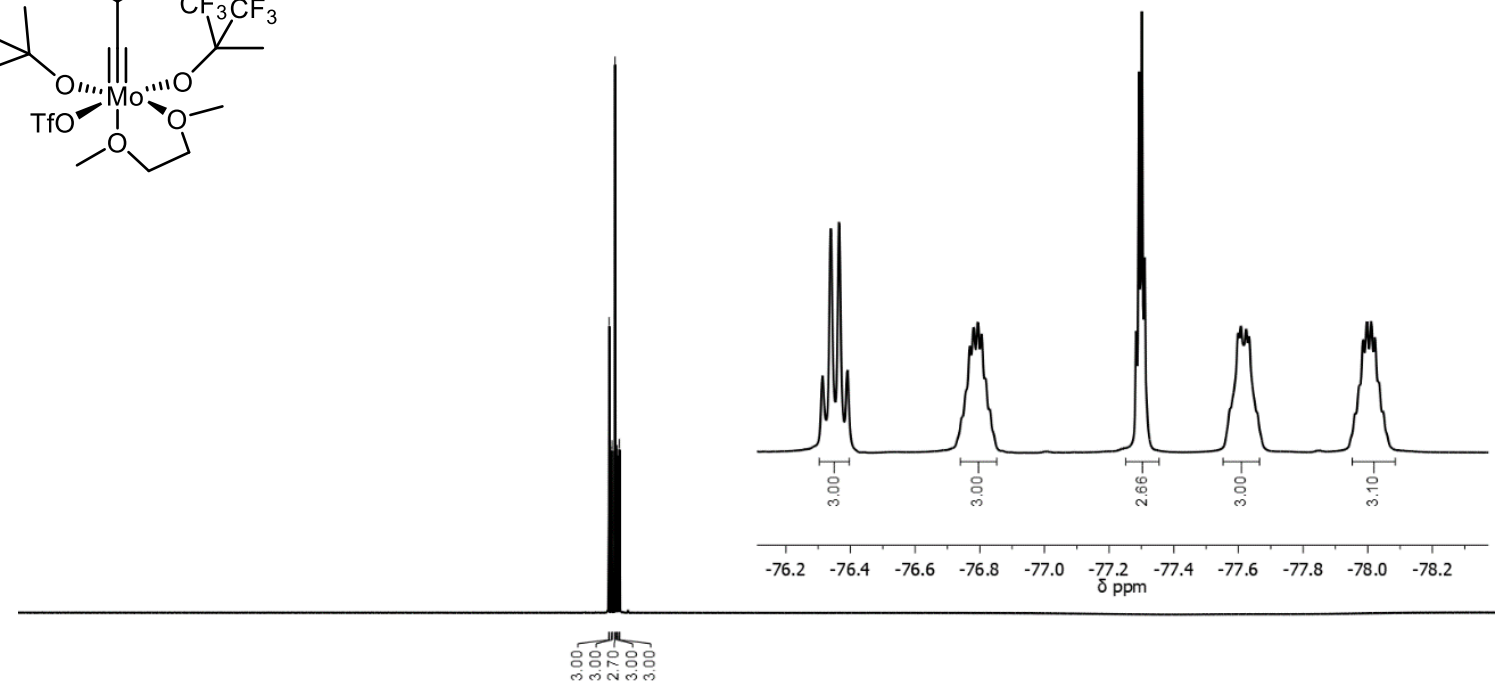

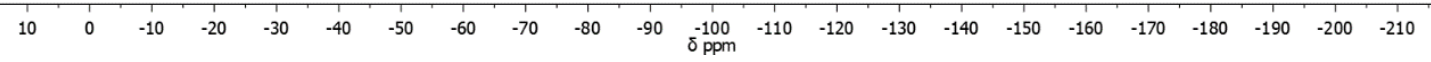

Figure S8: $\left.{ }^{19} \mathrm{~F} \mathrm{NMR} \mathrm{(375} \mathrm{MHz,} \mathrm{CDCl}_{3}\right)$ spectrum of Mo2. 
<smiles>COc1ccc(C(=O)OC(C)(OC(C)(C)C(F)(F)F)C(F)(F)F)cc1</smiles>
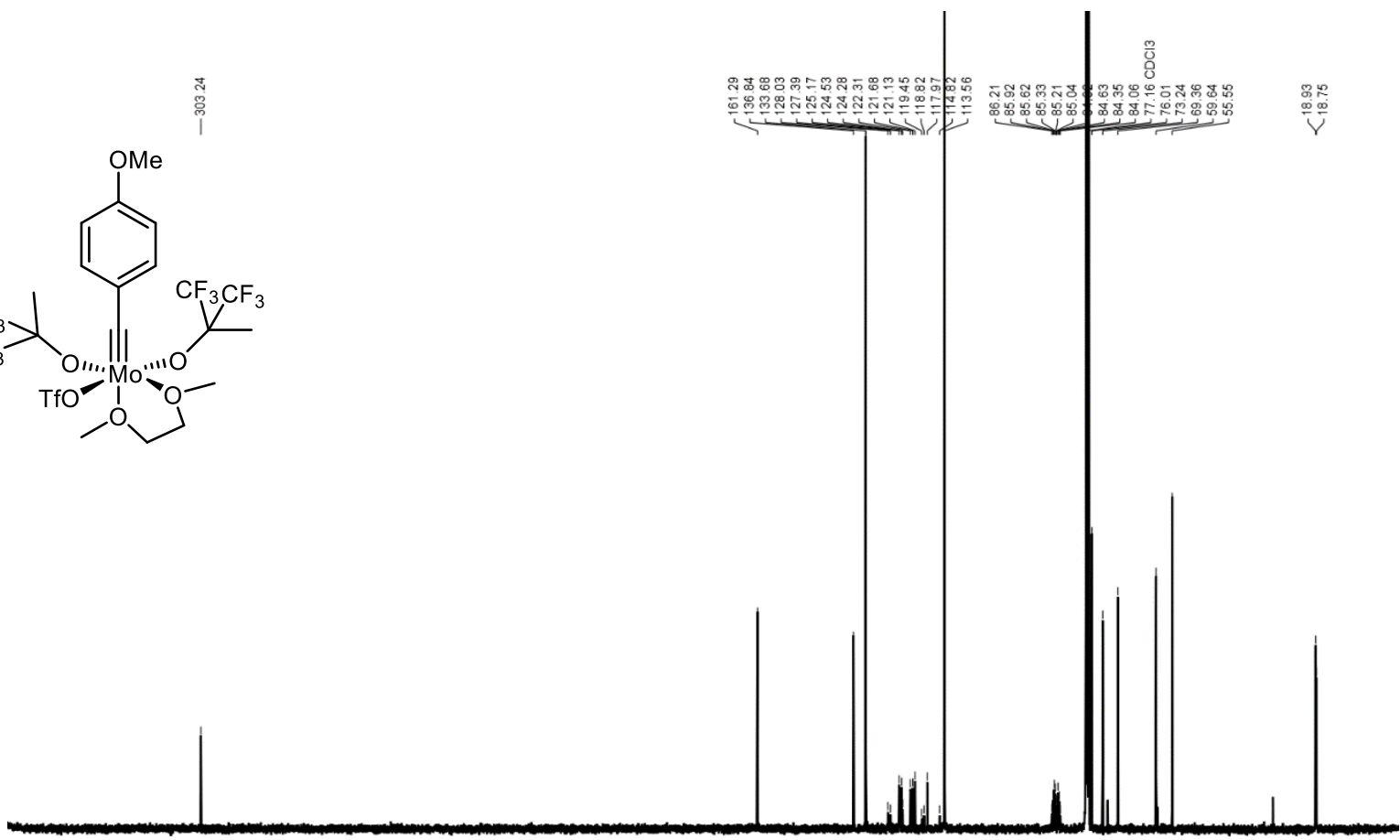

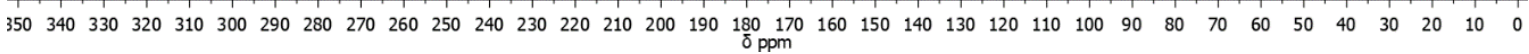

Figure S9: ${ }^{13} \mathrm{C}$ NMR (100 MHz, $\left.\mathrm{CDCl}_{3}\right)$ spectrum of $\mathbf{M o 2}$.

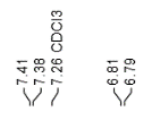

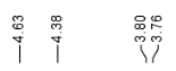

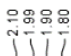

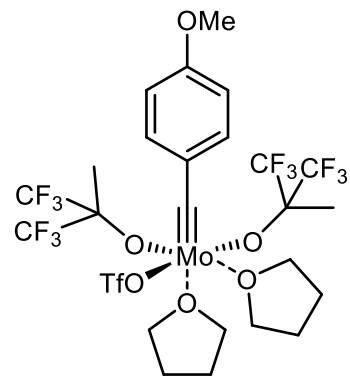

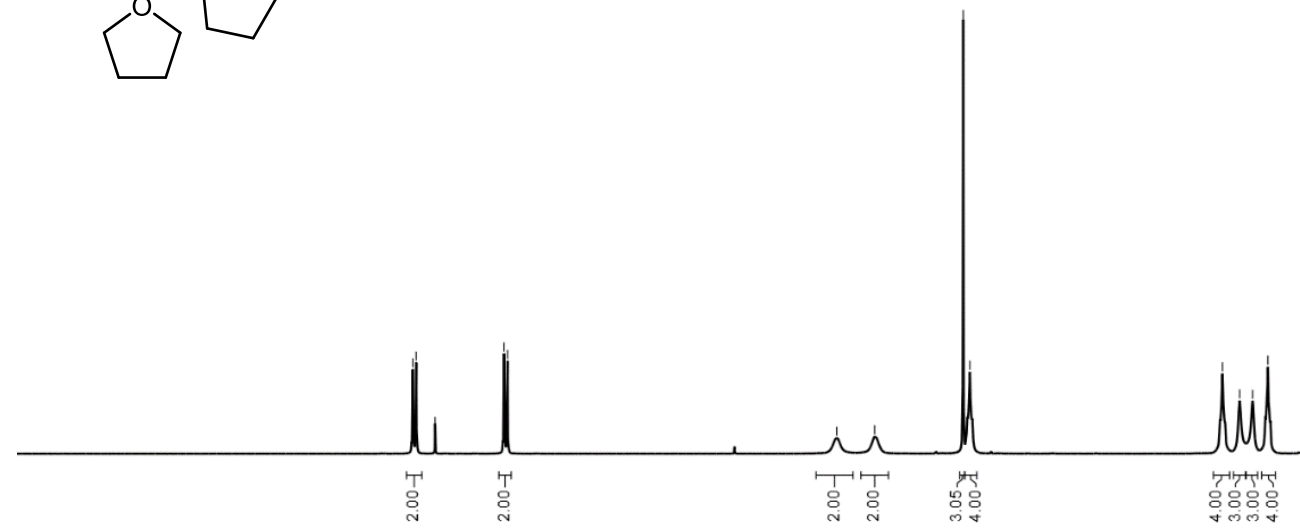

$\begin{array}{llll}9.5 & 9.0 & 8.5 & 8.0\end{array}$
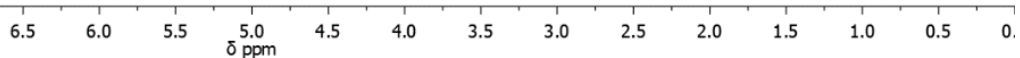

Figure S10: ${ }^{1} \mathrm{H}$ NMR $\left(400 \mathrm{MHz}, \mathrm{CDCl}_{3}\right)$ spectrum of Mo3. 


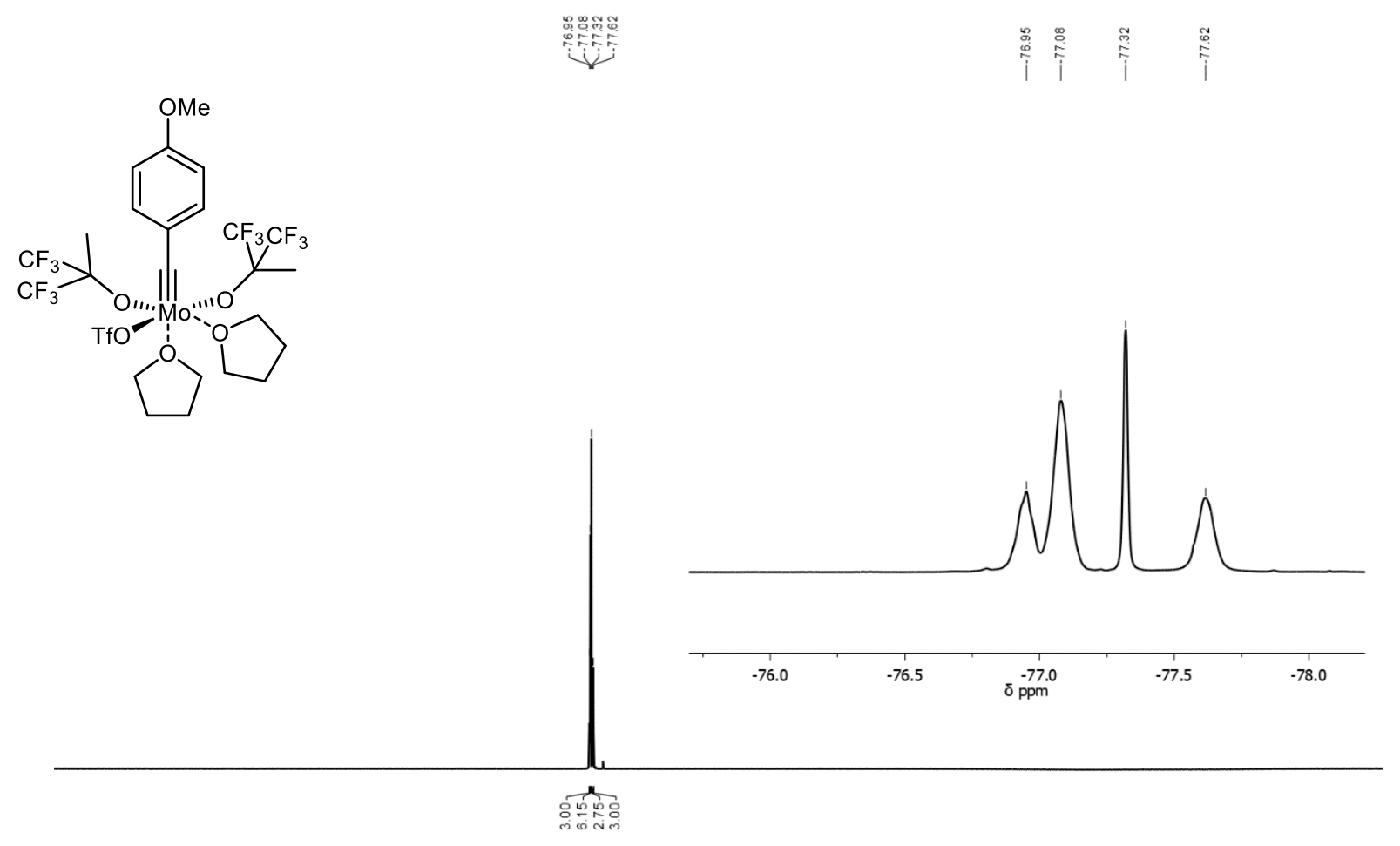

$\begin{array}{llllllllllllllllllllllll}10 & 0 & -10 & -20 & -30 & -40 & -50 & -60 & -70 & -80 & -90 & -100 & -110 & -120 & -130 & -140 & -150 & -160 & -170 & -180 & -190 & -200 & -210\end{array}$ Figure S11: ${ }^{19} \mathrm{C}$ NMR (375 MHz, $\left.\mathrm{CDCl}_{3}\right)$ spectrum of Mo3.
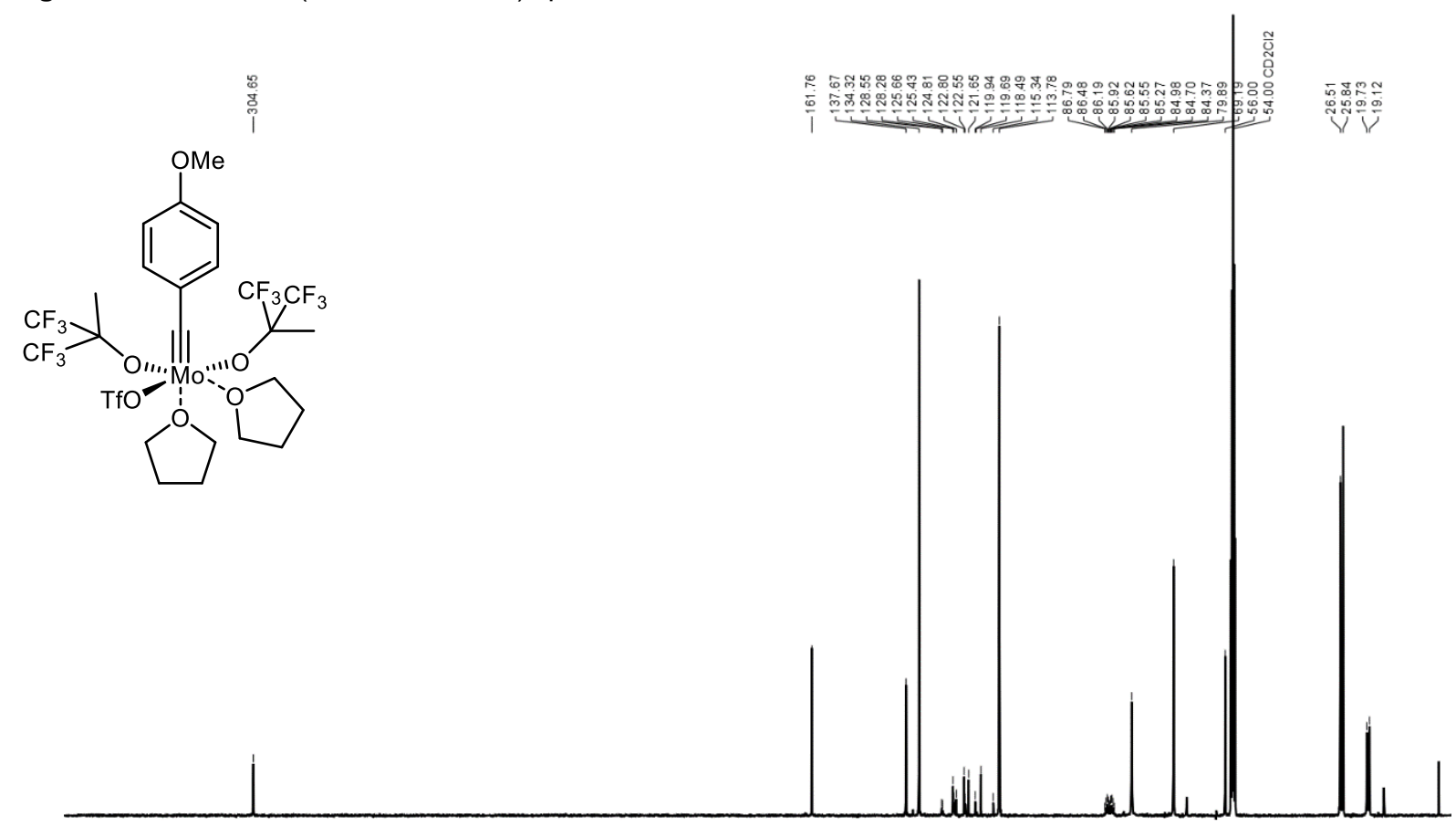

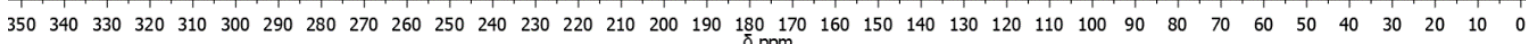
Figure S12: ${ }^{13} \mathrm{C}$ NMR (100 MHz, $\left.\mathrm{CD}_{2} \mathrm{Cl}_{2}\right)$ spectrum of Mo3. 


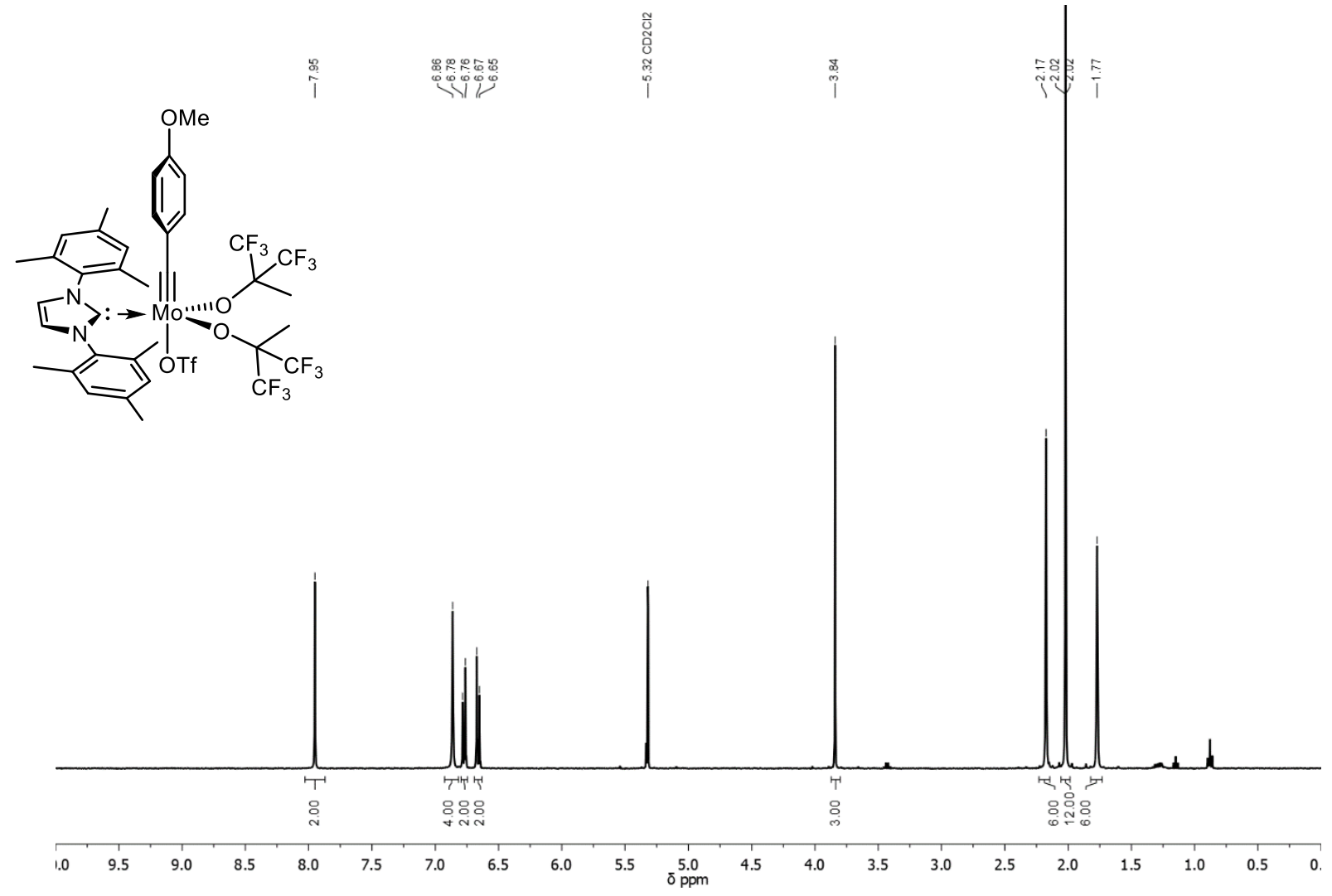

Figure S13: ${ }^{1} \mathrm{H}$ NMR $\left(400 \mathrm{MHz}, \mathrm{CD}_{2} \mathrm{Cl}_{2}\right)$ spectrum of Mo4.
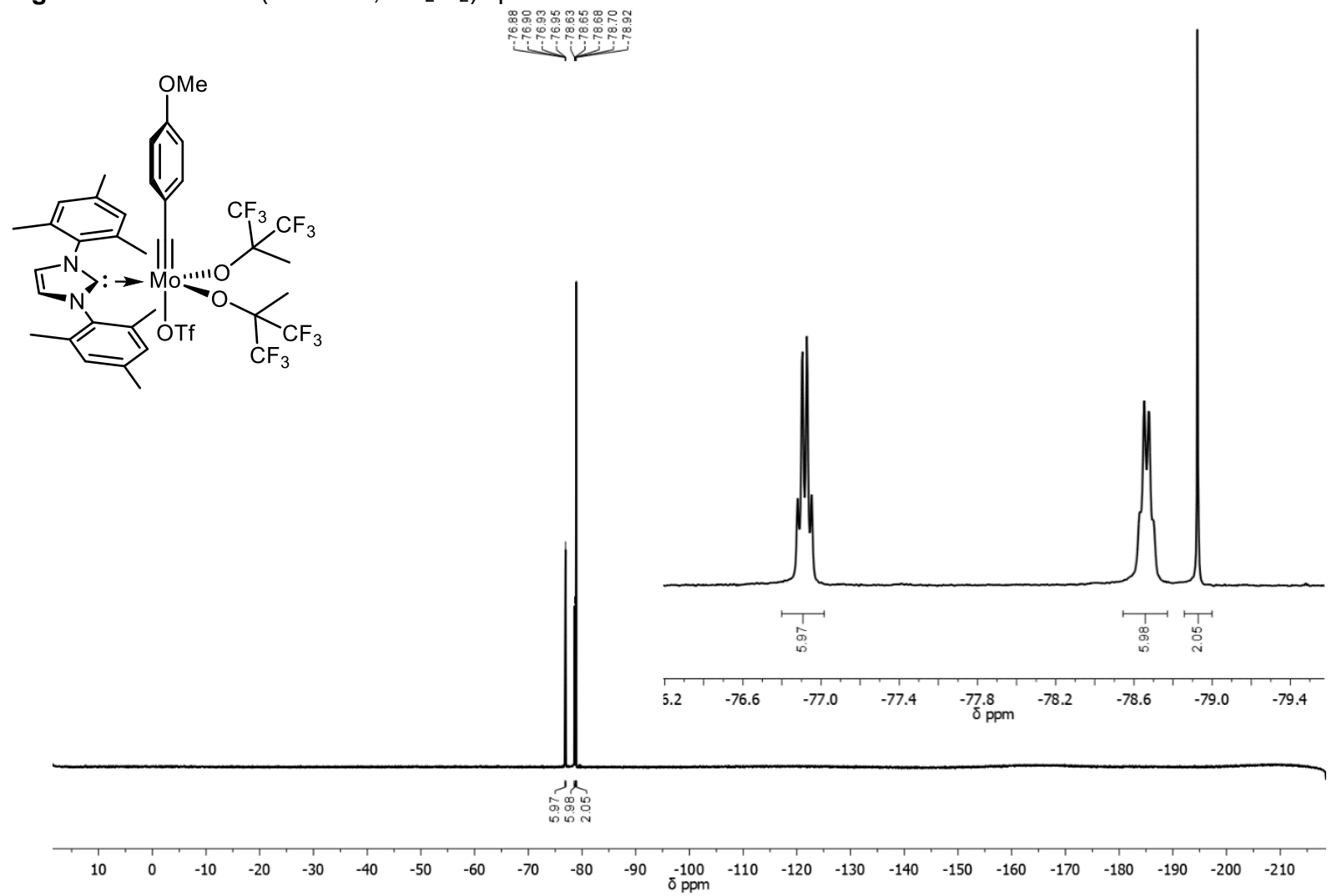

Figure S14: ${ }^{19} \mathrm{~F}$ NMR $\left(375 \mathrm{MHz}, \mathrm{CD}_{2} \mathrm{Cl}_{2}\right)$ spectrum of Mo4. 


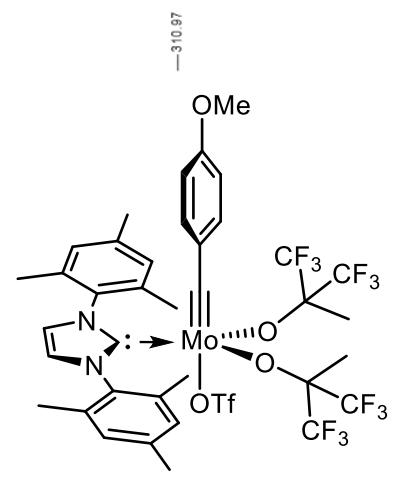

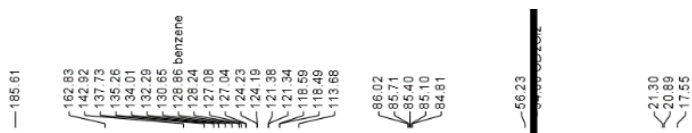

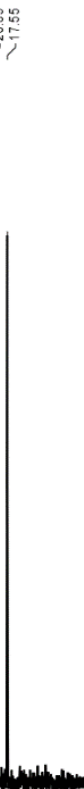

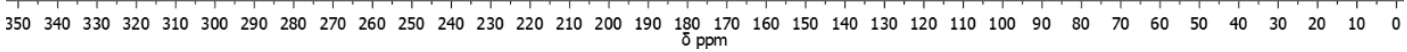

Figure S15: ${ }^{13} \mathrm{C}$ NMR (100 MHz, $\mathrm{CD}_{2} \mathrm{Cl}_{2}$ ) spectrum of Mo4.

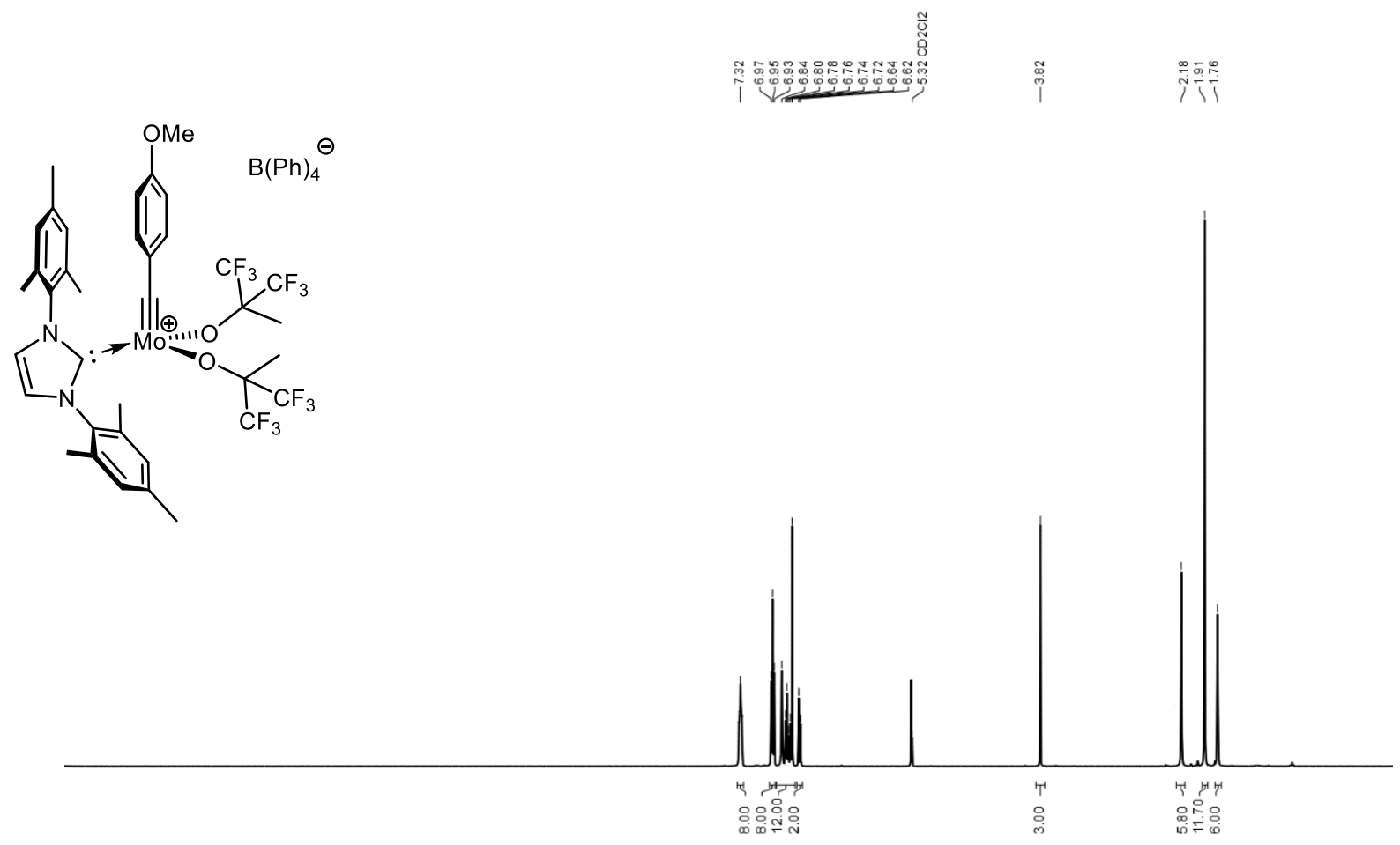

$\begin{array}{lllllllllllllllllllllllllllllllllllll}15.0 & 14.5 & 14.0 & 13.5 & 13.0 & 12.5 & 12.0 & 11.5 & 11.0 & 10.5 & 10.0 & 9.5 & 9.0 & 8.5 & 8.0 & 7.5 & 7.0 & 6.5 & 6.0 & 5.5 & 5.0 & 4.5 & 4.0 & 3.5 & 3.0 & 2.5 & 2.0 & 1.5 & 1.0 & 0.5 & 0.0\end{array}$

Figure S16: ${ }^{1} \mathrm{H}$ NMR (400 MHz, $\left.\mathrm{CD}_{2} \mathrm{Cl}_{2}\right)$ spectrum of Mo5. 

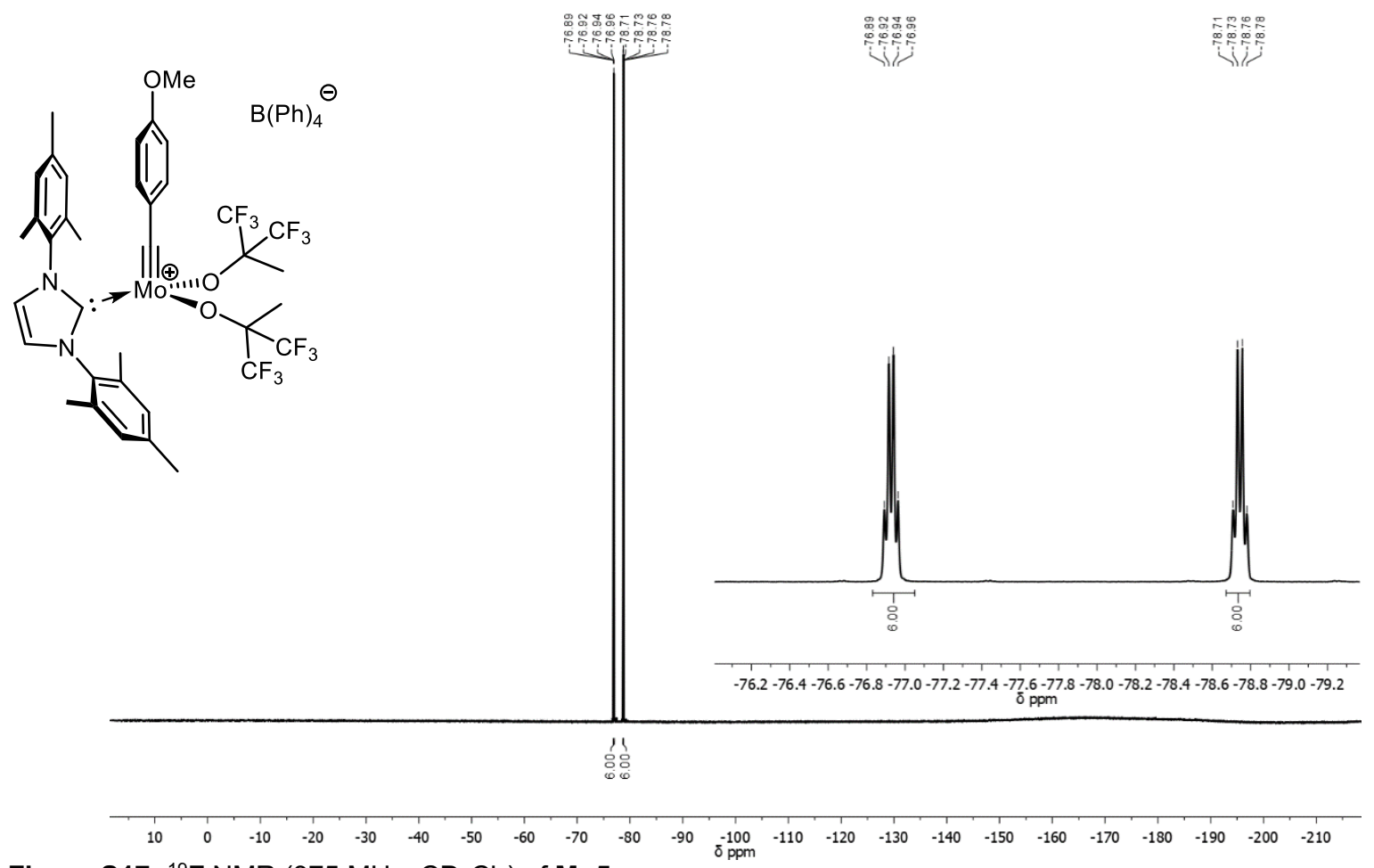

Figure S17: ${ }^{19} \mathrm{~F}$ NMR (375 MHz, $\mathrm{CD}_{2} \mathrm{Cl}_{2}$ ) of Mo5.
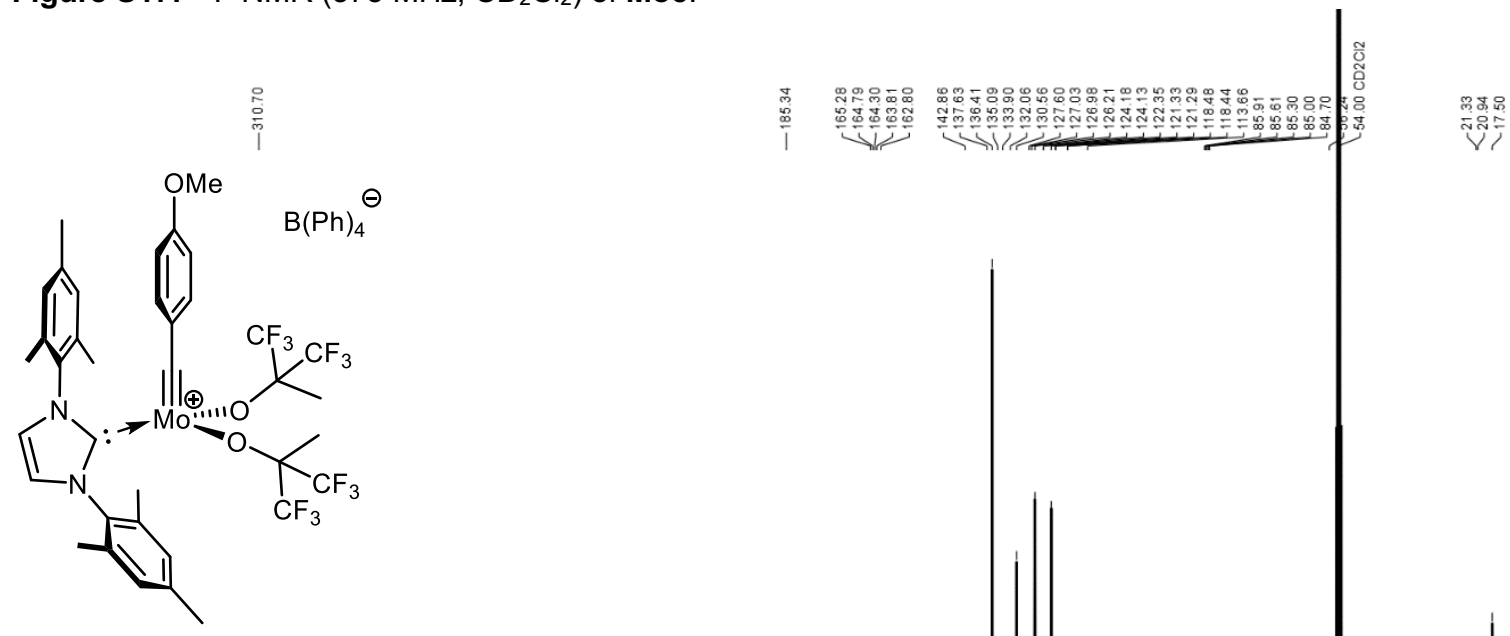

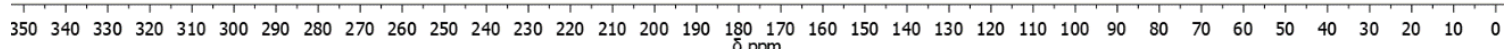
Figure S18: ${ }^{13} \mathrm{C}$ NMR (100 MHz, $\left.\mathrm{CD}_{2} \mathrm{Cl}_{2}\right)$ spectrum of Mo5. 


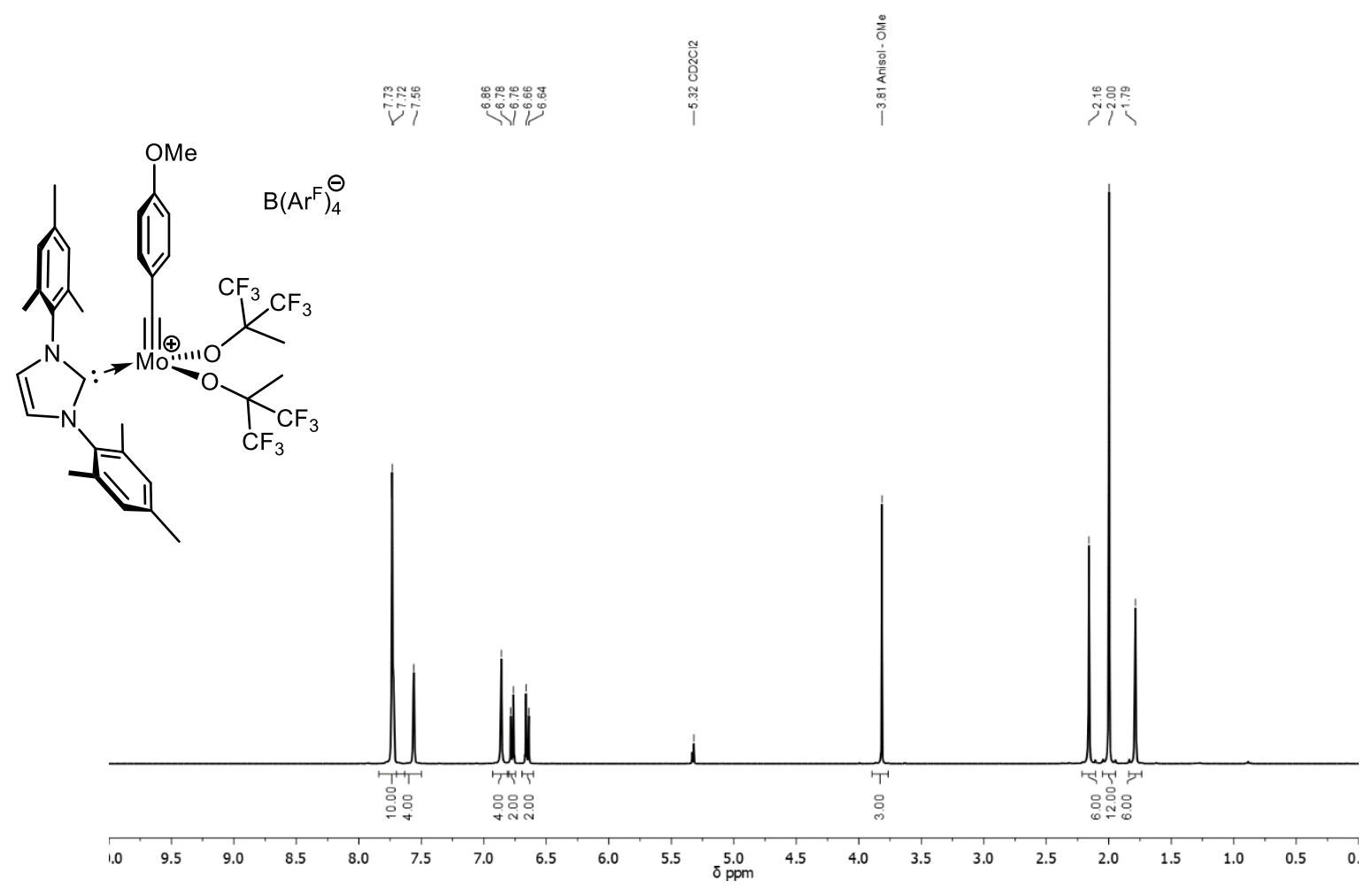

Figure S19: ${ }^{1} \mathrm{H}$ NMR $\left(400 \mathrm{MHz}, \mathrm{CD}_{2} \mathrm{Cl}_{2}\right)$ spectrum of Mo6.

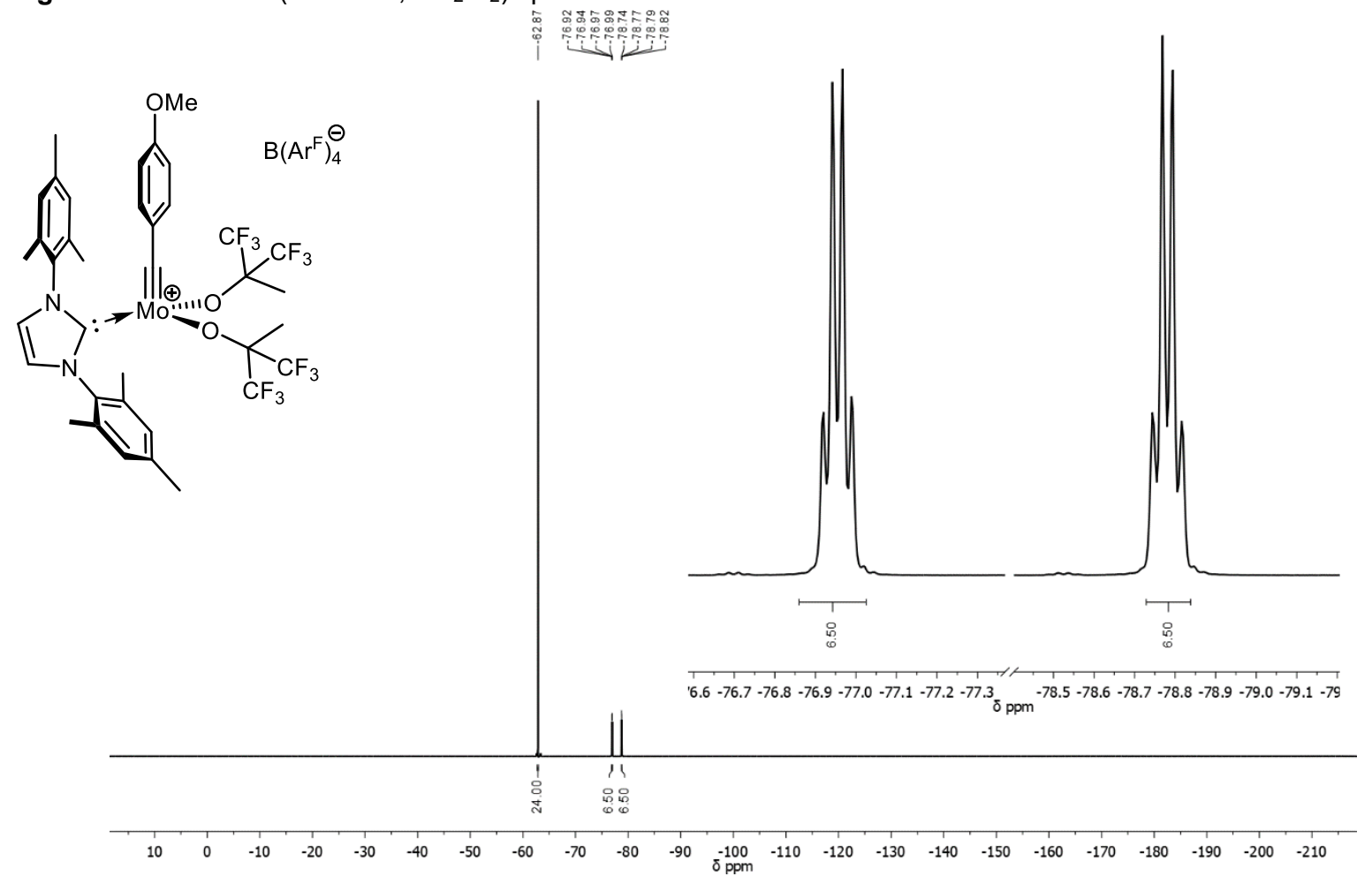

Figure S20: ${ }^{19} \mathrm{~F}$ NMR $\left(375 \mathrm{MHz}, \mathrm{CD}_{2} \mathrm{Cl}_{2}\right)$ spectrum of Mo6. 

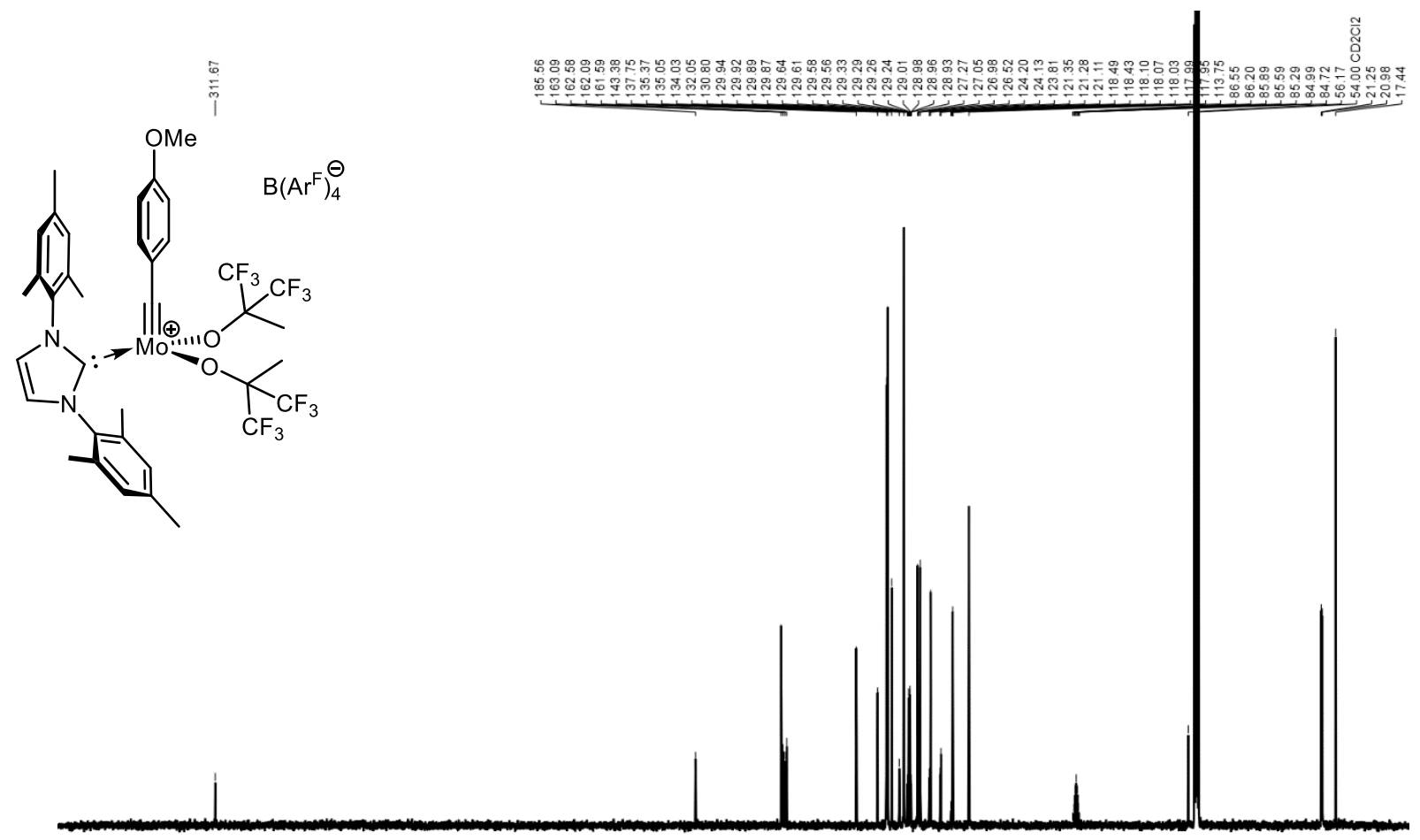

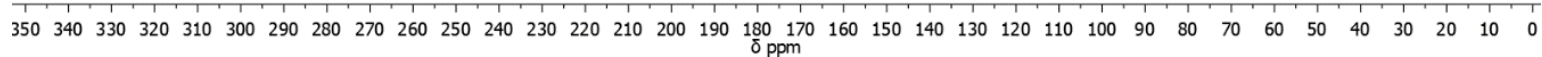

Figure S21: ${ }^{13} \mathrm{C}$ NMR $\left(100 \mathrm{MHz}, \mathrm{CD}_{2} \mathrm{Cl}_{2}\right)$ spectrum of Mo6.

\section{References}

1. Heppekausen, J.; Stade, R.; Goddard, R.; Fürstner, A., Practical New Silyloxy-Based Alkyne Metathesis Catalysts with Optimized Activity and Selectivity Profiles. J. Am. Chem. Soc. 2010, 132, 11045-11057. 